\title{
Mapping Autosomal Recessive Intellectual Disability: Combined Microarray and Exome Sequencing Identifies 26 Novel Candidate Genes in 192 Consanguineous Families
}

Ricardo Harripaul, MSc ${ }^{1,2}$, Nasim Vasli, $\mathrm{PhD}^{1}$, Anna Mikhailov, BSc ${ }^{1}$, Muhammad Arshad Rafiq, $\mathrm{PhD}^{1,3}$, Kirti Mittal, $\mathrm{PhD}^{1}$, Christian Windpassinger, $\mathrm{PhD}^{4}$, Taimoor I. Sheikh, MPhil ${ }^{1,2}$, Abdul Noor, $\mathrm{PhD}^{5,6}$, Huda Mahmood, $\mathrm{BSc}^{1}$, Samantha Downey ${ }^{1,7}$, Maneesha Johnson ${ }^{1,7}$, Kayla Vleuten $^{1,7}$, Lauren Bell ${ }^{1,7}$, Muhammad Ilyas, M.Phil ${ }^{8}$, Falak Sher Khan, MS ${ }^{9}$, Valeed Khan, $\mathrm{MS}^{9}$, Mohammad Moradi, MSc ${ }^{10}$, Muhammad Ayaz ${ }^{11}$, Farooq Naeem, PhD ${ }^{11,12}$, Abolfazl Heidari, $\mathrm{PhD}^{1,13}$, Iltaf Ahmed, $\mathrm{PhD}^{14}$, Shirin Ghadami, $\mathrm{PhD}^{15}$, Zehra Agha, $\mathrm{PhD}^{3}$, Sirous Zeinali, $\mathrm{PhD}^{15}$, Raheel Qamar, PhD ${ }^{3,16}$, Hossein Mozhdehipanah, $\mathrm{MD}^{17}$, Peter John, $\mathrm{PhD}^{14}$, Asif Mir, $\mathrm{PhD}^{8}$, Muhammad Ansar, $\mathrm{PhD}^{9}$, Leon French, $\mathrm{PhD}^{18}$, Muhammad Ayub, MBBS, MD ${ }^{11,12}$, John $\mathrm{B}$. Vincent, $\mathrm{PhD}^{1,2,19}$

${ }^{1}$ Molecular Neuropsychiatry \& Development (MiND) Lab, Campbell Family Mental Health Research Institute, Centre for Addiction and Mental Health, Toronto, ON, Canada;

${ }^{2}$ Institute of Medical Science, University of Toronto, Toronto, ON, Canada;

${ }^{3}$ Dept. of Biosciences, COMSATS Institute of Information Technology, Islamabad, Pakistan;

${ }^{4}$ Institute of Human Genetics, Medical University of Graz, Graz, Austria;

${ }^{5}$ Department of Pathology and Laboratory Medicine, Mount Sinai Hospital, Toronto, ON, Canada;

${ }^{6}$ Department of Laboratory Medicine and Pathobiology, University of Toronto, Toronto, ON, Canada; 
${ }^{7}$ Fleming College, Peterborough, ON, Canada;

${ }^{8}$ Human Molecular Genetics Lab, Department of Bioinformatics and Biotechnology, FBAS, International Islamic University, Islamabad, Pakistan;

${ }^{9}$ Department of Biochemistry, Quaid-i-Azam University, Islamabad, Pakistan;

${ }^{10}$ Qazvin University of Medical Science, Qazvin, Iran;

${ }^{11}$ Lahore Institute of Research \& Development, Lahore, Pakistan;

${ }^{12}$ Department of Psychiatry, Queen's University, Kingston, ON, Canada;

${ }^{13}$ Division of Hematology/Oncology, Hospital for Sick Children, Toronto, ON, Canada;

${ }^{14}$ Atta-ur-Rehman School of Applied Biosciences (ASAB), National University of Sciences and Technology (NUST), Islamabad, Pakistan;

${ }^{15}$ Department of Molecular Medicine, Biotechnology Research Center, Pasteur Institute of Iran, Tehran, Iran;

${ }^{16}$ Department of Biochemistry, Al-Nafees Medical College, Isra University, Islamabad, Pakistan;

${ }^{17}$ Department of Neurology, Qazvin University of Medical Sciences, Qazvin, Iran;

${ }^{18}$ Computational Neurobiology Lab, Campbell Family Mental Health Research Institute, Centre for Addiction and Mental Health, Toronto, ON, Canada;

${ }^{19}$ Department of Psychiatry, University of Toronto, Toronto, ON, Canada. 


\section{Corresponding Authors:}

Muhammad Ayub, MBBS, MD,

Department of Psychiatry,

752 King Street West,

Queen's University,

Kingston, ON K7L 7X3, Canada

(ma84@queensu.ca)

John B. Vincent, PhD

Neurogenetics Section, Campbell Family Mental Health Research Institute

Centre for Addiction and Mental Health (CAMH)

R-32, 250 College Street,

Toronto, ON M5T 1R8, Canada

(john.vincent@camh.ca) 
Approximately $1 \%$ of the global population is affected by intellectual disability (ID), and the majority receive no molecular diagnosis. Previous studies have indicated high levels of genetic heterogeneity, with estimates of more than 2500 autosomal ID genes, the majority of which are autosomal recessive (AR). Here, we combined microarray genotyping, homozygosity-by-descent (HBD) mapping, copy number variation (CNV) analysis, and whole exome sequencing (WES) to identify disease genes/mutations in 192 multiplex Pakistani and Iranian consanguineous families with non-syndromic ID. We identified definite or candidate mutations (or CNVs) in 51\% of families in 72 different genes, including 26 not previously reported for ARID. The new ARID genes include nine with loss-of-function mutations (ABI2, MAPK8, MPDZ, PIDD1, SLAIN1, TBC1D23, TRAPPC6B, UBA7, and USP44), and missense mutations include the first reports of variants in BDNF or TET1 associated with ID. The genes identified also showed overlap with de novo gene sets for other neuropsychiatric disorders. Transcriptional studies showed prominent expression in the prenatal brain. The high yield of AR mutations for ID indicated that this approach has excellent clinical potential and should inform clinical diagnostics, including clinical whole exome and genome sequencing, for populations in which consanguinity is common. As with other AR disorders, the relevance will also apply to outbred populations. 


\section{Introduction}

Approximately $1 \%$ of the global population is affected by intellectual disability (ID) ${ }^{1}$, which can have a devastating effect on the lives of the affected individuals and their families and is a major challenge at the clinical level. Genetic factors are involved in the aetiology of $25-50 \%$ of ID cases $^{2}$. The clinical presentation and aetiology of ID are complex and highly heterogeneous, thus leading to a poor rate of molecular diagnosis and inadequate clinical management and counselling.

ID can be divided into two groups: syndromic (S) ID, in which comorbid illness or physical features are present, and nonsyndromic (NS) ID, in which no such comorbidities are present. Of 700 known ID genes (S and NS), fewer than 50 genes are mutated in NS autosomal recessive ID (NS-ARID) ${ }^{2}$. X-linked ID may account for only $10-12 \%$ of ID cases $^{3}$. Dominant autosomal variants occurring de novo may contribute to a large proportion of sporadic cases, particularly in outbred populations ${ }^{4-6}$. Autosomal recessive (AR) variants also play a significant role in ID, because recessive variants can remain in the population in heterozygous form. Estimates have suggested that there may be more than 2500 autosomal ID genes in total-the majority being recessive ${ }^{7}$. In populations with high levels of consanguinity, most ID-causing mutations are recessive ${ }^{7}$. Even in outbred populations, $13-24 \%$ of ID cases have been estimated to be due to autosomal recessive causes ${ }^{7}$.

Despite an increased diagnostic yield among those who receive testing ${ }^{2}$, currently, the majority of individuals with ID receive no molecular diagnosis ${ }^{8}-$ a shortcoming that can affect health and lifespan. Recent studies have indicated that the median age of death is 13 years 
younger for males with ID and 20 years younger for females with ID than in the general population ${ }^{9}$. Although advances in genotyping and sequencing technology have accelerated the rate of gene discovery for $\mathrm{ID}^{10}$, the majority of ID genes remain undetected. However, largescale ID family studies are making significant inroads ${ }^{11}$. Homozygosity mapping has been proven to be an effective method for gene identification in consanguineous populations ${ }^{11-17}$. Consanguineous marriages lead to a marked increase in the frequency of severe recessive disorders $^{18}$. Collectively, countries with levels of consanguinity higher than $10 \%$, mainly in Africa, the Middle East and South Asia ${ }^{19,20}$, represent a population of $\sim 1.3$ billion. In Pakistan, $\sim 62.7 \%$ of the population engages in consanguineous marriages, of which $\sim 80.4 \%$ are firstcousin marriages ${ }^{21}$. The rate of consanguineous marriages in Iran is estimated at $40 \%^{22}$. Here, we present a study of multiplex ID families from Pakistan $(\mathrm{N}=176)$ and Iran $(\mathrm{N}=16)$ using microarray-based genotyping to identify autozygous regions (HBD shared by affected family members), coupled with whole exome sequencing (WES) to identify causal variants (see Figure 1 for workflow). In total, we identified single candidate genes/variants in 88 families (50\% were loss-of-function (LoF) mutations), and ten candidate pathogenic genomic variants (CNVs) in nine families.

\section{Methods}

\section{Family recruitment}

Institutional research ethics board consent was given for the study by the Centre for Addiction \& Mental Health, Toronto, as well as by the institutes at the recruiting sites (details in the Supplementary Methods). Families were recruited on the basis of diagnosis of ID in more than 
one individual (or ID and/or learning disability for $\mathrm{N}=13$ families; or both ID and psychosis within the family, $\mathrm{N}=5$ ) but with no obvious dysmorphic features or comorbidities and with parental consanguinity. Typically marriages were first- or second-cousin marriages; however, in a number of families, the exact relationship between the parents could not be established, but the marriage was within the same clan or caste. Written informed consent was obtained from all participants. Blood was drawn, and genomic DNA was extracted by standard methods. Cases of fragile X (tested using established methods ${ }^{23,24}$ ), Down's syndrome and other clearly recognizable syndromes were excluded. Summary statistics for the families are given in Table 1.

\section{Autozygosity Mapping}

Autozygosity mapping was performed using microarray data from the Illumina Human CoreExome, Affymetrix Mapping 500K Nspl, Affymetrix CytoScan HD and Affymetrix SNP 5.0 or 6.0. All genotyping was performed according to the manufacturer's protocol, and data were processed using either the Affymetrix Genotyping Console (500K and 5.0), Affymetrix ChAS Software Suite (CytoScan HD and SNP 6.0) or the Illumina GenomeStudio platform (Illumina CoreExome). All data were exported to PLINK format for analysis using HomozygosityMapper ${ }^{25}$ and FSuite ${ }^{26}$. Details are provided in the Supplementary Methods.

CNV Analysis

CNV analysis was performed to identify homozygous CNVs in HBD regions or heterozygous CNVs that could indicate cases of intra-familial genetic heterogeneity, which were then excluded from the HBD/WES analyses (details in the Supplementary Methods). 
Whole Exome Sequencing (WES)

WES was performed for one or more affected member from each family, using sequencing facilities at $\mathrm{CAMH}$, Toronto. Three different next-generation sequencing platforms were used across the study, taking advantage of newer platforms as they became available for research at CAMH (SOLiD 5500 platform (Life Technologies) for 49 families (51 individuals), using a protocol reported previously ${ }^{27}$; Ion Proton platform/Ion Ampliseq ${ }^{\mathrm{TM}}$ Exome kit (Life Technologies) for 49 individuals from 30 families; Illumina HiSeq2500 platform, using ThruPLEX DNA-seq 96D kit (Rubicon, R400407) and SureSelect XT2 Target Enrichment (Agilent Technologies) system for 150 families).

\section{Sequencing Alignment and Variant Calling}

We used an in-house pipeline to map and call variants on the different types of sequencing data for this study. The pipeline is summarized in Supplementary Figure 1 and in the Supplementary Methods.

After putative variants were identified, Sanger sequencing was used to validate the variants and determine whether the variant segregated with the disease by testing the parents and other affected and unaffected individuals within the family.

\section{Gene list construction}

We combined the genes listed in Supplementary Table 3 with other genes for X-linked or autosomal recessive intellectual disability/mental retardation in OMIM. This full list is available 
as Supplementary Table 7. This gene list was used in the pathway and gene expression analyses (details in the Supplementary Methods).

Database searches across different neuropsychiatric and neurodevelopmental disorders and functional and animal models

We compared our variants to several databases including EpilepsyGene, Gene2Phenotype, Gene2Cognition, Schizophrenia Genebook, published disease-specific gene-sets ${ }^{6}, \mathrm{HGMD}^{28}$, $\mathrm{OMIM}^{29}, \mathrm{Zfin}^{30}$, targets of FMRP through high-throughput sequencing of RNAs by cross-linking immune-precipitation (HITS-CLIP) ${ }^{31}$, and Mouseportal

(http://www.sanger.ac.uk/science/collaboration/mouse-resource-portal, accessed May 2016).

Searches were performed to identify genes/variants overlapping with our dataset.

\section{Results}

Our study identified single candidate genes/variants for 88 (81 autosomal recessive, 6 X-linked, and 1 de novo (heterozygous)) of the 192 families (Table $2 \& 3, \&$ Supplementary Table 3a). Twenty-six of the genes identified in this cohort have not previously been reported for NS-ARID. An additional eleven genes were previously first reported from this cohort $\left(C C 2 D 2 A^{32} ; T C T N 2^{33}\right.$; TRAPPC9 ${ }^{12} ;$ MAN1B1 $^{13} ;$ FBXO31 $^{27} ;$ METTL23 $^{14} ;$ FMN2 $^{15} ;$ DCPS $^{16} ;$ HMNT $^{17} ;$ NSUN2 $^{34} ;$ MBOAT7 $\left.^{351}\right)$. Most of these genes have since been reported in multiple ID families, including in outbred populations (e.g., TRAPPC9 ${ }^{36} ; M A N 1 B 1^{37}$ ). Likely pathogenic $C N V$ s were identified in at least nine families (not including the VPS13B and TUSC3 deletions in AN51 and AN21, respectively). 
Homozygous Loss-of-Function Mutations

Homozygous truncating LoF mutations were found as single candidate variants in 43 families, including nine genes that had not been previously described in relation to NS-ARID: $A B I 2$, MAPK8, MPDZ, PIDD1, SLAIN1, TBC1D23, TRAPPC6B, UBA7, and USP44. None of these genes have been noted as tolerant to loss of function through WES of 3,222 British adults of Pakistani $\operatorname{origin}^{38}$

We identified the same nonsense mutation in PIDD1 in two unrelated Pakistani families (ASMR105 and ASMR110; Figure 2). PIDD1 encodes p53-Induced Death Domain Protein (PIDD; MIM 605247), and GIn863* disrupts the death domain (DD), through which PIDD1 interacts with other DD proteins such as RIP1 or CRADD/RAIDD. Truncating mutations in CRADD have previously been reported for NS-ARID (MRT34 ${ }^{39}$ ), and thus our findings support the involvement of PIDD-related pathways in the aetiology of ID.

Homozygous truncating mutations in TRAPPC9 have been reported for NS-ARID ${ }^{12,36,40-}$

${ }^{43}$; thus it is notable that we identified a nonsense mutation in TRAPPC6B, which encodes a second member of the same protein trafficking particle complex.

A nonsense mutation was identified in SLAIN1 that segregated fully in family PK68 (Figure 2). SLAIN1 and SLAIN2 encode microtubule plus-end tracking proteins that have been shown to be crucial for normal axonal growth in developing hippocampal neurons ${ }^{44}$.

We report here a homozygous nonsense mutation in the gene UBA7 in family PK34. UBA7 encodes ubiquitin-activating enzyme 7 , which is believed to be involved in the ubiquitin 
conjugation pathway ${ }^{45}$. Family PK34 is one of three families in the study that did not meet the criteria for ID and instead were reported as having learning disorders and were considered relatively high functioning. We also report that this variant is present at a relatively high frequency in the South Asian population ( $M A F=0.0054$; ExAC database), and two homozygotes for this variant were among the control group. Thus, this variant and gene may be a risk factor for a much milder form of cognitive disability and thus may potentially be present in the control South Asian population $(\mathrm{N}>8,000)$ used in the ExAC database.

We also identified a LoF mutation in TMEM135, which has previously reported in a large Iranian NS-ARID cohort by Najmabadi et al, 2011 (in which a missense mutation, Cys228Ser, was reported) ${ }^{11}$, and in Wright et al, 2014 (missense) ${ }^{46}$, as a quantitative trait locus associated with intelligence ${ }^{47}$.

\section{Missense mutations}

We identified homozygous missense variants as single candidate variants in 43 families, including 16 for which the identified genes have not previously been reported for NS-ARID (AFF3, BDNF, CAPS, DMBT1, DUOX2, EXTL3, FBXO47, LAMC1, MAP3K7, SDK2, SPATA13, SUMF2, SYNRG, TET1, VPS35, and ZBTB11). Although LoF mutations are frequently more convincing than missense mutations, a number of the homozygous missense changes reported here are of particular interest, owing to the known nature or function of the protein or the likely effect of the amino acid substitution on protein function. For instance, we report a homozygous missense change Met122Thr in the gene for brain-derived neurotrophic factor, BDNF, which has been implicated in many studies of neuropsychiatric disorders ${ }^{48}$ and is a known gene target 
of $M E C P 2$, the Rett syndrome gene. ${ }^{49}, 50$ This variant replaces a methionine, a large, non-polar residue with an S-methyl thioether side chain, that is fully conserved across the vertebrate lineage with threonine, a small, polar residue with a hydroxyl side chain. This variant is present with a frequency of 0.0001647 in ExAC (in South Asians MAF=0.001211) with no homozygotes and has not previously been reported in any publications. The BDNF protein is important for the survival, differentiation and development of neurons in the central nervous system (CNS). Hence, the identification of Met122Thr in connection with cognitive disability is likely to be of great interest, and functional studies of the effects of Met122Thr on BDNF function are warranted.

We detected a homozygous Lys2056Asn change in TET1 in family PK70 that is not present in any variant or mutation databases. Methylcytosine dioxygenase TET proteins play a role in the DNA methylation process and gene activation, and TET1 is an important regulator of neuronal differentiation. TET1 has been implicated in schizophrenia (SCZ) and bipolar disorder (BD) by down-regulation of the expression of GAD1, RELN, and BDNF genes through epigenetic mechanisms in the prefrontal cortex and in the cerebellum in autism ${ }^{51,52}$. Significant upregulation of TET1 mRNA in the parietal cortex of psychosis patients has also been reported $^{53}$. Tet1 knockout results in impaired hippocampal neurogenesis, cognitive deficits in mice $^{54}$ and abnormal brain morphology in zebrafish ${ }^{55}$. The large basic, charged, hydrophobic lysine residue at this position is conserved in mammals (or as glutamic acid in non-mammalian vertebrates) and is replaced in this variant by the small polar asparagine residue. 
The homozygous His880GIn change identified in the zinc finger/BTB domain gene ZBTB11 in family AN50 disrupts a canonical $\mathrm{Zn}^{2+}$-binding residue in one of the zinc fingers and is likely to result in an alteration in the specificity of DNA-binding/gene regulation. AFF3 is an autosomal homolog of the X-linked ID gene AFF2 (MRX-FRAXE; MIM 309547). As with AFF2, AFF3 is also associated with a fragile site (FRA2A) in which expansion of a CGG trinucleotide repeat triggers hypermethylation and gene silencing in association with neurodevelopmental disorders ${ }^{56}$. The Gly1215Val variant identified here is located within a five-amino acid Cterminal motif (GIn-Gly-Leu-His-Trp) that is highly conserved across the vertebrate lineage.

In family AS70, a missense mutation was identified in exon 12 of the gene MAP3K7. Although missense mutations in this gene have recently been reported for frontometaphyseal dysplasia $^{57}$ (FMD) and cardiospondylocarpofacial syndrome (CSCF syndrome) ${ }^{58}$, members of family AS70 have no skeletal dysplasia or obvious dysmorphic features, thus suggesting even greater pleiotropy of this gene. We note that although the Arg410GIn mutation identified is located in exon 12, which is alternatively spliced and present in both transcripts $B$ and $C$ but neither A nor D, all MAP3K7 mutations reported to date for FMD or CSCF syndrome are located in canonical exons (i.e., in all four transcript variants). Furthermore, analysis of mouse RNAseq data from the ENCODE UW project (through UCSC Genome Browser) suggests that although exon 12 is expressed in many adult tissues, including the brain, it is absent from skeletal muscle.

Families with multiple variants 
For eleven additional families, between two and four putative damaging variants were identified after filtration that fulfilled the criteria and segregated in the families; however, without functional evidence in support of pathogenicity, it is not possible to narrow these variants to a single candidate (Supplementary Table 3B).

\section{X-linked variants}

A number of the families were compatible for both AR and X-linkage, and several variants or CNVs on the $\mathrm{X}$ chromosome were identified, including two variants in ATRX and a 6.7-Mb interstitial duplication on Xp22.31-p22.2.

We report a missense mutation, Ser57Pro, in the X-chromosomal gene MAGEA11, segregating in family PK87. A GIn4Arg variant in MAGEA11 has recently been reported among a cohort of X-linked ID families ${ }^{59}$. MAGEA11 shows protein interaction with TRMT1-also reported here for NS-ARID-as determined by yeast-two-hybrid analysis ${ }^{60-62}$.

In another large multiplex and multi-branch family, the missense mutation Arg190His of the $\mathrm{X}$-linked MECP2 gene is present in hemizygous form in a single male with mild ID and in heterozygous form in several females with mild ID or mild ID with psychosis or depression. Interestingly, for the female heterozygotes with ID, all appeared cognitively normal until the age of $\sim 9$ years, when cognitive regression started, and for the single male hemizygote, cognitive regression began much earlier, at $<5$ years of age. The family also has several males with SCZ, with onset at 18 years but without cognitive regression amounting to ID, who are wild type for this variant (see Supplementary Figure 2). The Arg190 residue is a critical DNA- 
binding amino acid within an AT-hook domain, and a de novo mutation at this residue, Arg190Cys, has been reported in a SCZ patient ${ }^{63}$.

Genes with 'hits' in multiple families

Confidence in gene discovery relies on the identification of multiple affected families with mutations in the same gene and/or replication in further studies. However, owing to the anticipated high degree of genetic heterogeneity in NS-ARID, large sample sizes are typically required to include multiple families with mutations in the same gene. Comparison across different studies is thus vital. Of the ARID genes reported here for the first time, several are conspicuous because of the presence of mutations in multiple families. In our study, the same nonsense mutation in PIDD1 was observed in two apparently unrelated Pakistani families. We also provide confirmatory families/mutations for recently reported ARID genes such as C12ORF4, CCDC82, MBOAT7, IMPA1, TMEM135, PGAP2, GPT2 (2 families), TDP2 (2 families), and GMPPA (Table 3, and Supplementary Table 3). In addition, mutations in the gene for IDassociated brain malformation polymicrogyria, GPR56, are present in three families in this study and thus may represent a relatively large proportion of ID families in these populations.

Families with mutations in previously identified genes for metabolic or hormonal disorders

We report three mutations in the thyroid dyshormonogenesis (TDH) genes TPO (thyroid peroxidase), TG (thyroglobulin) ${ }^{64}$ ), and DUOX2 (thyroid oxidase 2). With adequate clinical resources available in most developed countries, many of these cases would likely have been diagnosed, and in some cases, for example, those with mutations in $\mathrm{N}$-acetylglutamate synthase (NAGS), TPO, TG and DUOX2, early treatment would have prevented ID development. 
Given the prevalence of mutations in these genes in a relatively modest number of families, it is likely that these disorders are relatively common causes of ID in populations in which consanguinity is common, but access to clinical diagnostics is poor. Mutations in GNE have previously been linked with sialuria (dominant; MIM 269921)-an extremely rare metabolic disorder-and Nonaka myopathy (recessive; MIM 605820), whereas here we report a homozygous missense mutation in a family with only ID and no myopathy. Although we were unable to perform biochemical analysis of this family, we anticipate that this discovery may represent a previously unreported recessive form of sialuria.

\section{Copy number variation (CNV) analysis}

In addition to HBD analysis, the microarray data were used for CNV analysis: first, to identify possible intra-familial genetic heterogeneity with large, probably pathogenic heterozygous loss/gain CNVs, and second, to detect homozygous genic CNVs within mapped HBD regions. For the former, several candidate pathogenic CNVs were identified (Supplementary Table 4), including an 8.4 Mb deletion of 2q14.1-q14.3 (chr2:116583565-124954598) in one of the two affected individuals in family PK117. Deletions within this region have previously been reported for autism spectrum disorder ${ }^{65}$ and in a patient with a mild holoprosencephaly spectrum phenotype ${ }^{66}$. An overlapping but slightly proximal deletion ( chr2: 114188161-119321989) has been reported to be compatible with a normal phenotype ${ }^{67}$. In one family, PK28, all three affected males in one branch were shown to have a large (6.7 $\mathrm{Mb}$ ) interstitial duplication spanning cytobands Xp22.31-p22.2. 
For homozygous CNVs in HBD regions, we identified homozygous deletions in known ARID genes in several families (Table 3 and Supplementary Table 3), including a $170 \mathrm{~kb}$ homozygous deletion spanning 9 of 10 exons of the NS-ARID gene TUSC3 in family AN21 ${ }^{68}$ and a $51 \mathrm{~kb}$ deletion spanning exons 37 to 40 of the Cohen syndrome gene, VPS13B, in family AN51 ${ }^{69}$. Homozygous disrupting loss CNVs included a $50 \mathrm{~kb}$ deletion spanning exons 2 and 3 of $R A B 8 B$ in PK95-7; however, this deletion was not in a shared HBD region and was thus considered a potential case of intra-familial genetic heterogeneity.

Databases for neuropsychiatric disorders

Genes relevant for ID are also frequently identified in individuals with autism spectrum disorders (ASD) and epilepsy (both of which frequently also present with ID). In addition, there is growing support for overlap of ID genes with genes affecting other neuropsychiatric disorders $^{70}$. For example, genes such as NRXN1 and ANK3, which have been linked with SCZ, BD and ASD by CNV and/or genome-wide association studies, are also known ARID genes (NRXN1: PTHLS2, MIM 614325; ANK3: MRT37, MIM 615494). For this reason, we attempted to evaluate the genes identified here in variant databases or among lists of genes associated with such disorders. We screened various epilepsy or neuropsychiatric disease-specific databases or published datasets for the presence of variants in these genes. In the SCZ/control exome sequence database Genebook ${ }^{71,72}$, none of the identified variants in Table 2 were present. However, for several of the genes, an increased burden of rare variants has been reported in SCZ cases versus controls ( $\mathrm{p}<0.05$ for VPS35, SYNRG, DMBT1, ALPI, and NEU4; $\mathrm{p}<0.002$ for 
SLC13A5), although when corrected for multiple testing, no individual gene-based tests achieved statistical significance.

In epilepsy databases, we identified ATRX, MECP2, SLC13A5, and ST3GAL3, all of which have been reported in cases of epilepsy as well as ID. These four genes are either inherited as autosomal recessive or $\mathrm{X}$-linked recessive. ATRX and MECP2 interact with each other and are involved in chromatin binding and gene regulation ${ }^{73}$, and mutations in either gene can have diverse effects on DNA methylation patterns and brain development.

\section{Protein interaction analysis}

We used BioGrid (http://thebiogrid.org) to identify protein interactors for each of the 67 different ARID genes identified among our population (Supplementary Table 3A) as well as GRIN2B (Supplementary Table 3C). Interacting proteins are listed, along with gene ontology processes, functions, and cellular compartments, in Supplementary Table 5.

\section{Pathway analyses}

In agreement with past findings of genetic heterogeneity, we observed limited overlap with the gene ontology gene sets. Of the 14,312 sets tested, 16 survived multiple test correction (qvalue $<0.05$ ), and a few top-ranked terms are of interest (Supplementary Table 6). Protein glycosylation, which is known to be associated with ID, was ranked 6th with 9 overlapping genes (corrected $\mathrm{p}<0.05$ ). As mentioned above, TPO, DUOX2, and TG are known to be involved in thyroid hormone generation (rank: 14 , corrected $p<0.05$ ). The smoothened signalling pathway was ranked 32nd with 4 overlapping genes (NDST1, CC2D2A, TCTN2, and BBS7). Anatomical expression analyses 
With the exception of the spinal cord, all neural tissues were enriched in the expression of the ID genes (corrected $p<0.0001$, Supplementary Table 8 ). The frontal cortex was the most enriched, with 1.4 times the expected number of expressed genes. Although the brain expressed a majority of all genes, the ID genes appeared to show specificity. Of the 17 foetal tissues tested, the female gonad and testis had the highest number of expressed genes but did not show enriched expression of the ID genes (corrected $p>0.12$ ).

\section{Developmental brain expression analyses}

Across the developmental transcriptome, ID genes were expressed at higher levels in the normal prenatal brain (Figure 3). The amygdaloid complex showed the most consistent enrichment (5 specimens with significantly higher expression of ID genes). Foetal brain samples from 21 and 24 weeks post-conception showed the highest number of regions with significant expression (> 4 or more). By contrast, enriched expression was not observed in the postnatal brain samples. This prenatal pattern found in exon microarray expression profiles was also observed in RNA sequencing measurements in a largely overlapping set of samples from the same resource (BrainSpan, Supplementary Figure 3,). The RNA sequencing measurements showed donor-specific patterns with many enriched regions. These global patterns were not consistent across donors of the same age, thus suggesting that the RNA sequencing data may have normalization artefacts not present in the microarray measurements. Grouping the ID gene list into genes associated with glycosylation or hormones and metabolism revealed aboveaverage prenatal expression for all groupings (Figure 3). Genes with metabolic or hormonalassociated function had the most stable trajectory. We also observed higher expression for 
those groups in the prenatal brain in the BrainCloud ${ }^{74}$ resource, which assayed the prefrontal cortex across human development (Supplementary Figure 4).

\section{Discussion}

This study describes a cohort of 192 multiplex ID families from Pakistan and Iran. Using combined microarray genotyping, HBD) mapping, CNV analysis, and WES, we identified definite or candidate mutations (or likely pathogenic CNVs) in $51 \%$ of families in 72 different genes, including 26 not previously reported for ARID.

Notably, $50 \%$ of the variants that we report as single probable mutations were LoF changes. This result compares well with findings from an earlier study reported by Najmabadi et al, $2011^{11}$, in which 50 new genes for NS-ARID were reported, of which $40 \%$ had LoF mutations. In general, LoF mutations provide a higher degree of confidence of disease association than missense mutations.

There are a number of likely reasons that genes/mutations were not found for some families: 1. intra-familial etiologic heterogeneity; 2 . poor WES depth of coverage at the etiologic gene/mutation; 3. intronic or intergenic causative mutations, which are not detected by WES. Whole genome sequencing may address this issue; 4 . Common variants have been reported in association with many complex diseases, including traits such as intelligence or cognitive ability $^{75,76}$. It is plausible that a proportion of ID cases with familial aggregation are caused by variants, possibly interacting, that are common in the general population but have low penetrance. These variants together could potentially cause ID; however, this hypothesis has 
not been explored in ID. 5. Non-genetic factors may be prevalent in some families, e.g., prenatal or perinatal insult ${ }^{77}$.

Given that there may be an overlap in the genetic aetiology of neurodevelopmental and neuropsychiatric disorders, we cross-referenced our ID gene list with those from studies of other neuropsychiatric/neurodevelopmental disorders. Many genes identified for ARID have been implicated across disorders (see Figure 4). For instance, of the newly identified genes, SLAIN1 has been listed as one of the top-ranked genes for the burden of variants among a large cohort with SCZ $(N=1,392)^{78}$. Data from the Autism Sequencing Consortium ${ }^{79}$ have revealed de novo LoF mutations in SPATA13 and TBC1D23, as well as de novo missense mutations in $A B I 2$, TET1, and SYNRG. Li et al $(2016)^{80}$ reported variants in both MPDZ and SPATA13 in ASD cohorts and a de novo missense variant in MAPK8 in $\mathrm{SCZ}^{72}$. In addition, whereas heterozygous (typically de novo) mutations in SCN1A have been linked with ASD, ID, and epileptic encephalopathy, the missense SCN1A variant reported here is homozygous. A number of ARID genes (e.g., NRXN1, CNTNTAP2, and ANK3) have already been implicated in neuropsychiatric disorders by GWAS or CNV studies (as heterozygous). We speculate that, in addition to pleiotropy with other neuropsychiatric/neurodevelopmental disorders, the mode of inheritance may also be variable, (e.g., SPATA13: homozygous missense variant in ID (this study) and de novo LoF variant in $\mathrm{ASD}^{80} ; \mathrm{HNMT}$ : homozygous missense variant in $\mathrm{ID}^{17}$ and heterozygous splice mutation in $\mathrm{SCZ}$ (Genebook)). ID may be conceptualized as a much more severe form of neurodevelopmental disorder than ASD and SCZ. 
Transcriptional analysis of our gene set combined with known ARID genes revealed greater levels of transcription in the prenatal brain than the postnatal or adult brain (Figure 3A) and, in particular, higher levels in the frontal cortex, hippocampus and amygdala (Figure 3B). ID is primarily a disorder of brain development, and thus it was reassuring to observe relevant patterns of spatiotemporal expression of ID genes. Pathway analysis of the gene set showed several significant pathways; thyroid metabolism was prominent, as was protein glycosylation and the Smoothened signalling pathway. This and other studies have predicted involvement of numerous different pathways in ID, which is probably a reflection of the high genetic heterogeneity in ID.

Our findings and those of other groups studying the genetics of NS-ARID should be invaluable for the development of targeted sequencing gene panels for diagnostic screening and for the development of clinical whole exome and whole genome sequencing. In this regard, both the replication of previous findings and new discoveries in this study are important. Replication enhances the confidence in the validity of findings, and new discoveries provide opportunities for further exploration of the functions and biological pathways of the newly associated genes. Cumulatively, such studies have also mapped genes across the human genome in which LoF mutations are viable, and the roles of these genes in human development are thus likely to be amenable to further study and comparison with similar mutations in model organisms. Furthermore, a more comprehensive picture is being assembled of the molecular components and mechanisms that are important for the development of a fully functioning central nervous system, as well as the points in the mechanisms that are most vulnerable to genetic mutation. Through comparison with similar studies, we also note that many more such 
discoveries are needed to complete the picture. The ultimate challenge, to devise targeted therapeutic strategies for ID patients, is thus a step closer.

The discovery of disease-causing mutations in consanguineous families immediately creates opportunities for carrier screening among relatives and prevention of ID, thus providing a direct benefit to the families, communities, and to public health. In addition, the identification of genetic causes for ID allows individuals with ID to be subgrouped on the basis of gene or pathway. This categorization can lead to the development of cohorts that can then be studied prospectively for the natural course of the disease and health complications and that can also be targeted for therapeutic strategies, thus representing a step towards personalized medicine in this important clinical population.

\section{Acknowledgements}

We thank the study participants and their families for their invaluable contributions to this study. We also thank Justin Foong and Andy Wang for their assistance in setting up the NGS analysis pipeline on the CAMH SCC. Computations were performed on the CAMH Specialized Computing Cluster. The SCC is funded by The Canada Foundation for Innovation and the Research Hospital Fund. We also acknowledge the assistance of Muhammad Aslam (LIRD), Tanveer Nasr (Mayo Hospital, Lahore and Chaudhry Hospital, Gujranwala, Pakistan), Muhammad Ilyas (International Islamic University, Islamabad, Pakistan), Reza Najafipour and Soraya Keshavarz (Qazvin University of Medical Science), and Ali Rashidi-Nezhad (Tehran University of Medical Sciences) for family recruitment and phenotyping. We also thank Drs 
Hans van Bokhoven and Arjen de Brower (Radboud University Medical Center, Nijmegen, The

Netherlands), who provided microarray SNP data for families ZA5 and ZA17. RH was supported

by a Peterborough K.M. Hunter Charitable Foundation Graduate Scholarship. This study was

partially supported by a grant from the Canadian Institutes of Health Research to JBV (\#MOP-

102758).

\section{Conflict of Interest}

The authors report no conflicts of interest.

Supplementary information is available at the Molecular Psychiatry website.

\section{References}

1. Maulik PK, Mascarenhas MN, Mathers CD, Dua T, Saxena S. Prevalence of intellectual disability: a meta-analysis of population-based studies. Research in developmental disabilities 2011; 32(2): 419-436.

2. Vissers LE, Gilissen C, Veltman JA. Genetic studies in intellectual disability and related disorders. Nature Reviews Genetics 2015.

3. Ropers H-H, Hamel BC. X-linked mental retardation. Nature Reviews Genetics 2005; 6(1): 46-57.

4. de Ligt J, Willemsen MH, van Bon BW, Kleefstra T, Yntema HG, Kroes T et al. Diagnostic exome sequencing in persons with severe intellectual disability. New England Journal of Medicine 2012; 367(20): 1921-1929.

5. Hamdan FF, Srour M, Capo-Chichi J-M, Daoud H, Nassif C, Patry L et al. De novo mutations in moderate or severe intellectual disability. PLoS Genet 2014; 10(10): e1004772. 
6. Gilissen C, Hehir-Kwa JY, Thung DT, van de Vorst M, van Bon BW, Willemsen MH et al. Genome sequencing identifies major causes of severe intellectual disability. Nature 2014.

7. Musante L, Ropers HH. Genetics of recessive cognitive disorders. Trends in Genetics 2014; 30(1): 32-39.

8. Rauch A, Hoyer J, Guth S, Zweier C, Kraus C, Becker C et al. Diagnostic yield of various genetic approaches in patients with unexplained developmental delay or mental retardation. American journal of medical genetics Part A 2006; 140(19): 2063-2074.

9. Heslop P, Blair PS, Fleming P, Hoghton M, Marriott A, Russ L. The Confidential Inquiry into premature deaths of people with intellectual disabilities in the UK: a population-based study. The Lancet 2014; 383(9920): 889-895.

10. Yang Y, Muzny DM, Reid JG, Bainbridge MN, Willis A, Ward PA et al. Clinical whole-exome sequencing for the diagnosis of mendelian disorders. New England Journal of Medicine 2013; 369(16): 1502-1511.

11. Najmabadi H, Hu H, Garshasbi M, Zemojtel T, Abedini SS, Chen W et al. Deep sequencing reveals 50 novel genes for recessive cognitive disorders. Nature 2011; 478(7367): 57-63.

12. Mir A, Kaufman L, Noor A, Motazacker MM, Jamil T, Azam M et al. Identification of mutations in TRAPPC9, which encodes the NIK-and IKK- $\beta$-binding protein, in nonsyndromic autosomalrecessive mental retardation. The American Journal of Human Genetics 2009; 85(6): 909-915.

13. Rafiq MA, Kuss AW, Puettmann L, Noor A, Ramiah A, Ali G et al. Mutations in the alpha 1, 2mannosidase gene, MAN1B1, cause autosomal-recessive intellectual disability. The American Journal of Human Genetics 2011; 89(1): 176-182.

14. Bernkopf M, Webersinke G, Tongsook C, Koyani CN, Rafiq MA, Ayaz M et al. Disruption of the methyltransferase-like 23 gene METTL23 causes mild autosomal recessive intellectual disability. Human molecular genetics 2014; 23(15): 4015-4023.

15. Law R, Dixon-Salazar T, Jerber J, Cai N, Abbasi AA, Zaki MS et al. Biallelic truncating mutations in FMN2, encoding the actin-regulatory protein formin 2, cause nonsyndromic autosomal-recessive intellectual disability. The American Journal of Human Genetics 2014; 95(6): 721-728.

16. Ahmed I, Buchert R, Zhou M, Jiao X, Mittal K, Sheikh TI et al. Mutations in DCPS and EDC3 in autosomal recessive intellectual disability indicate a crucial role for mRNA decapping in neurodevelopment. Human molecular genetics 2015; 24(11): 3172-3180. 
17. Heidari A, Tongsook C, Najafipour R, Musante L, Vasli N, Garshasbi M et al. Mutations in the histamine N-methyltransferase gene, HNMT, are associated with nonsyndromic autosomal recessive intellectual disability. Human molecular genetics 2015; 24(20): 5697-5710.

18. Modell B, Darr A. Genetic counselling and customary consanguineous marriage. Nature Reviews Genetics 2002; 3(3): 225-229.

19. Hamamy H. Consanguineous marriages. Journal of community genetics 2012; 3(3): 185-192.

20. Saadat M. Consanguinity associated with child and adult mortality in 24 Asian and African countries, an ecological study. Iranian Journal of Public Health 2007; 36: 35-39.

21. Hussain R, Bittles A. The prevalence and demographic characteristics of consanguineous marriages in Pakistan. Journal of biosocial science 1998; 30(02): 261-275.

22. Najmabadi H, Motazacker MM, Garshasbi M, Kahrizi K, Tzschach A, Chen W et al. Homozygosity mapping in consanguineous families reveals extreme heterogeneity of non-syndromic autosomal recessive mental retardation and identifies 8 novel gene loci. Human genetics 2007; 121(1): 43-48.

23. Haddad LA, Mingroni-Netto RC, Vianna-Morgante AM, Pena SD. A PCR-based test suitable for screening for fragile $X$ syndrome among mentally retarded males. Human genetics $1996 ; 97(6)$ : 808-812.

24. Gazal S, Sahbatou M, Babron M-C, Génin E, Leutenegger A-L. FSuite: exploiting inbreeding in dense SNP chip and exome data. Bioinformatics 2014; 30(13): 1940-1941.

25. Seelow D, Schuelke M, Hildebrandt F, Nürnberg P. HomozygosityMapper-an interactive approach to homozygosity mapping. Nucleic acids research 2009; 37(suppl 2): W593-W599.

26. Gazal S, Sahbatou M, Babron MC, Genin E, Leutenegger AL. FSuite: exploiting inbreeding in dense SNP chip and exome data. Bioinformatics 2014; 30(13): 1940-1941.

27. Mir A, Sritharan K, Mittal K, Vasli N, Araujo C, Jamil T et al. Truncation of the E3 ubiquitin ligase component $\mathrm{FBXO} 31$ causes non-syndromic autosomal recessive intellectual disability in a Pakistani family. Human genetics 2014; 133(8): 975-984.

28. Stenson PD, Ball EV, Mort M, Phillips AD, Shiel JA, Thomas NS et al. Human gene mutation database (HGMD $\left.{ }^{\circledR}\right)$ : 2003 update. Human mutation 2003; 21(6): 577-581. 
29. Hamosh A, Scott AF, Amberger JS, Bocchini CA, McKusick VA. Online Mendelian Inheritance in Man (OMIM), a knowledgebase of human genes and genetic disorders. Nucleic acids research 2005; 33(suppl 1): D514-D517.

30. Bradford Y, Conlin T, Dunn N, Fashena D, Frazer K, Howe DG et al. ZFIN: enhancements and updates to the Zebrafish Model Organism Database. Nucleic acids research 2011; 39(suppl 1): D822-D829.

31. Darnell JC, Van Driesche SJ, Zhang C, Hung KYS, Mele A, Fraser CE et al. FMRP stalls ribosomal translocation on mRNAs linked to synaptic function and autism. Cell 2011; 146(2): 247-261.

32. Noor A, Windpassinger C, Patel M, Stachowiak B, Mikhailov A, Azam M et al. CC2D2A, encoding a coiled-coil and $C 2$ domain protein, causes autosomal-recessive mental retardation with retinitis pigmentosa. The American Journal of Human Genetics 2008; 82(4): 1011-1018.

33. Sang L, Miller JJ, Corbit KC, Giles RH, Brauer MJ, Otto EA et al. Mapping the NPHP-JBTS-MKS protein network reveals ciliopathy disease genes and pathways. Cell 2011; 145(4): 513-528.

34. Khan MA, Rafiq MA, Noor A, Hussain S, Flores JV, Rupp V et al. Mutation in NSUN2, which encodes an RNA methyltransferase, causes autosomal-recessive intellectual disability. The American Journal of Human Genetics 2012; 90(5): 856-863.

35. Johansen A, Rosti RO, Musaev D, Sticca E, Harripaul R, Zaki M et al. Mutations in MBOAT7, Encoding Lysophosphatidylinositol Acyltransferase I, Lead to Intellectual Disability Accompanied by Epilepsy and Autistic Features. The American Journal of Human Genetics 2016.

36. Marangi G, Leuzzi V, Manti F, Lattante S, Orteschi D, Pecile V et al. TRAPPC9-related autosomal recessive intellectual disability: report of a new mutation and clinical phenotype. European Journal of Human Genetics 2013; 21(2): 229-232.

37. Van Scherpenzeel M, Timal S, Rymen D, Hoischen A, Wuhrer M, Hipgrave-Ederveen A et al. Diagnostic serum glycosylation profile in patients with intellectual disability as a result of MAN1B1 deficiency. Brain 2014; 137(4): 1030-1038.

38. Narasimhan VM, Hunt KA, Mason D, Baker CL, Karczewski KJ, Barnes MR et al. Health and population effects of rare gene knockouts in adult humans with related parents. Science 2016; 352(6284): 474-477.

39. Puffenberger EG, Jinks RN, Sougnez C, Cibulskis K, Willert RA, Achilly NP et al. Genetic mapping and exome sequencing identify variants associated with five novel diseases. PloS one 2012; 7(1): e28936. 
40. Mochida GH, Mahajnah M, Hill AD, Basel-Vanagaite L, Gleason D, Hill RS et al. A truncating mutation of TRAPPC9 is associated with autosomal-recessive intellectual disability and postnatal microcephaly. The American Journal of Human Genetics 2009; 85(6): 897-902.

41. Philippe O, Rio M, Carioux A, Plaza J-M, Guigue P, Molinari F et al. Combination of linkage mapping and microarray-expression analysis identifies NF-KB signaling defect as a cause of autosomal-recessive mental retardation. The American Journal of Human Genetics 2009; 85(6): 903-908.

42. Jamra RA, Wohlfart S, Zweier M, Uebe S, Priebe L, Ekici A et al. Homozygosity mapping in 64 Syrian consanguineous families with non-specific intellectual disability reveals 11 novel loci and high heterogeneity. European Journal of Human Genetics 2011; 19(11): 1161-1166.

43. Kakar N, Goebel I, Daud S, Nürnberg G, Agha N, Ahmad A et al. A homozygous splice site mutation in TRAPPC9 causes intellectual disability and microcephaly. European journal of medical genetics 2012; 55(12): 727-731.

44. van der Vaart B, Franker MA, Kuijpers M, Hua S, Bouchet BP, Jiang K et al. Microtubule plus-end tracking proteins SLAIN1/2 and ch-TOG promote axonal development. The Journal of Neuroscience 2012; 32(42): 14722-14728.

45. Figueiredo T, Melo U, Pessoa A, Nobrega P, Kitajima J, Rusch $\mathrm{H}$ et al. A homozygous loss-offunction mutation in inositol monophosphatase 1 (IMPA1) causes severe intellectual disability. Molecular psychiatry 2015.

46. Wright CF, Fitzgerald TW, Jones WD, Clayton S, McRae JF, Van Kogelenberg M et al. Genetic diagnosis of developmental disorders in the DDD study: a scalable analysis of genome-wide research data. The Lancet 2015; 385(9975): 1305-1314.

47. Franić S, Groen-Blokhuis MM, Dolan CV, Kattenberg MV, Pool R, Xiao X et al. Intelligence: shared genetic basis between Mendelian disorders and a polygenic trait. European Journal of Human Genetics 2015.

48. Autry AE, Monteggia LM. Brain-derived neurotrophic factor and neuropsychiatric disorders. Pharmacological reviews 2012; 64(2): 238-258.

49. Chen WG, Chang Q, Lin Y, Meissner A, West AE, Griffith EC et al. Derepression of BDNF transcription involves calcium-dependent phosphorylation of MeCP2. Science 2003; 302(5646): 885-889. 
50. Martinowich $\mathrm{K}$, Hattori D, Wu H, Fouse S, He F, Hu Y et al. DNA methylation-related chromatin remodeling in activity-dependent BDNF gene regulation. Science 2003; 302(5646): 890-893.

51. Dong E, Ruzicka W, Grayson D, Guidotti A. DNA-methyltransferase1 (DNMT1) binding to CpG rich GABAergic and BDNF promoters is increased in the brain of schizophrenia and bipolar disorder patients. Schizophrenia research 2015; 167(1): 35-41.

52. Zhubi A, Chen Y, Dong E, Cook E, Guidotti A, Grayson D. Increased binding of MeCP2 to the GAD1 and RELN promoters may be mediated by an enrichment of 5-hmC in autism spectrum disorder (ASD) cerebellum. Translational psychiatry 2014; 4(1): e349.

53. Dong E, Gavin DP, Chen Y, Davis J. Upregulation of TET1 and downregulation of APOBEC3A and $A P O B E C 3 C$ in the parietal cortex of psychotic patients. Translational psychiatry 2012; 2(9): e159.

54. Zhang R-R, Cui Q-Y, Murai K, Lim YC, Smith ZD, Jin S et al. Tet1 regulates adult hippocampal neurogenesis and cognition. Cell Stem Cell 2013; 13(2): 237-245.

55. Li C, Lan Y, Schwartz-Orbach L, Korol E, Tahiliani M, Evans T et al. Overlapping Requirements for Tet2 and Tet3 in Normal Development and Hematopoietic Stem Cell Emergence. Cell reports 2015; 12(7): 1133-1143.

56. Metsu S, Rooms L, Rainger J, Taylor MS, Bengani H, Wilson DI et al. FRA2A is a CGG repeat expansion associated with silencing of AFF3. PLoS Genet 2014; 10(4): e1004242.

57. Wade EM, Daniel PB, Jenkins ZA, Mclnerney-Leo A, Leo P, Morgan T et al. Mutations in MAP3K7 that Alter the Activity of the TAK1 Signaling Complex Cause Frontometaphyseal Dysplasia. The American Journal of Human Genetics 2016; 99(2): 392-406.

58. Le Goff C, Rogers C, Le Goff W, Pinto G, Bonnet D, Chrabieh M et al. Heterozygous Mutations in MAP3K7, Encoding TGF- $\beta$-Activated Kinase 1, Cause Cardiospondylocarpofacial Syndrome. The American Journal of Human Genetics 2016; 99(2): 407-413.

59. Hu H, Haas S, Chelly J, Van Esch H, Raynaud M, de Brouwer A et al. X-exome sequencing of 405 unresolved families identifies seven novel intellectual disability genes. Molecular psychiatry 2016; 21(1): 133-148.

60. Rual J-F, Venkatesan K, Hao T, Hirozane-Kishikawa T, Dricot A, Li N et al. Towards a proteomescale map of the human protein-protein interaction network. Nature 2005; 437(7062): 11731178. 
61. Rolland T, Taşan M, Charloteaux B, Pevzner SJ, Zhong Q, Sahni N et al. A proteome-scale map of the human interactome network. Cell 2014; 159(5): 1212-1226.

62. Yu H, Tardivo L, Tam S, Weiner E, Gebreab F, Fan C et al. Next-generation sequencing to generate interactome datasets. Nature methods 2011; 8(6): 478-480.

63. McCarthy SE, Gillis J, Kramer M, Lihm J, Yoon S, Berstein Y et al. De novo mutations in schizophrenia implicate chromatin remodeling and support a genetic overlap with autism and intellectual disability. Molecular psychiatry 2014; 19(6): 652.

64. Mittal K, Rafiq MA, Rafiullah R, Harripaul R, Ali H, Ayaz $M$ et al. Mutations in the genes for thyroglobulin and thyroid peroxidase cause thyroid dyshormonogenesis and autosomalrecessive intellectual disability. Journal of Human Genetics 2016.

65. Pagnamenta AT, Bacchelli E, de Jonge MV, Mirza G, Scerri TS, Minopoli F et al. Characterization of a family with rare deletions in CNTNAP5 and DOCK4 suggests novel risk loci for autism and dyslexia. Biological psychiatry 2010; 68(4): 320-328.

66. Kevelam SH, van Harssel JJ, van der Zwaag B, Smeets HJ, Paulussen AD, Lichtenbelt KD. A patient with a mild holoprosencephaly spectrum phenotype and heterotaxy and a $1.3 \mathrm{Mb}$ deletion encompassing GLI2. American Journal of Medical Genetics Part A 2012; 158(1): 166-173.

67. Barber JC, Maloney VK, Bewes B, Wakeling E. Deletions of 2q14 that include the homeobox engrailed 1 (EN1) transcription factor are compatible with a normal phenotype. European journal of human genetics 2006; 14(6): 739-743.

68. Khan MA, Rafiq MA, Noor A, Ali N, Ali G, Vincent JB et al. A novel deletion mutation in the TUSC3 gene in a consanguineous Pakistani family with autosomal recessive nonsyndromic intellectual disability. BMC medical genetics 2011; 12(1): 56.

69. Rafiq MA, Leblond CS, Saqib MA, Vincent AK, Ambalavanan A, Khan FS et al. Novel VPS13B Mutations in Three Large Pakistani Cohen Syndrome Families Suggests a Baloch Variant with Autistic-Like Features. BMC medical genetics 2015; 16(1): 41.

70. Hall J, Owen MJ. Psychiatric classification-a developmental perspective. The British Journal of Psychiatry 2015; 207(4): 281-282.

71. Purcell SM, Moran JL, Fromer M, Ruderfer D, Solovieff N, Roussos P et al. A polygenic burden of rare disruptive mutations in schizophrenia. Nature 2014; 506(7487): 185-190. 
72. Fromer M, Pocklington AJ, Kavanagh DH, Williams HJ, Dwyer S, Gormley P et al. De novo mutations in schizophrenia implicate synaptic networks. Nature 2014; 506(7487): 179-184.

73. Nan X, Hou J, Maclean A, Nasir J, Lafuente MJ, Shu X et al. Interaction between chromatin proteins MECP2 and ATRX is disrupted by mutations that cause inherited mental retardation. Proceedings of the National Academy of Sciences 2007; 104(8): 2709-2714.

74. Colantuoni C, Lipska BK, Ye T, Hyde TM, Tao R, Leek JT et al. Temporal dynamics and genetic control of transcription in the human prefrontal cortex. Nature 2011; 478(7370): 519-523.

75. Clarke T, Lupton M, Fernandez-Pujals A, Starr J, Davies G, Cox S et al. Common polygenic risk for autism spectrum disorder (ASD) is associated with cognitive ability in the general population. Molecular psychiatry 2015.

76. Davies G, Marioni RE, Liewald DC, Hill WD, Hagenaars SP, Harris SE et al. Genome-wide association study of cognitive functions and educational attainment in UK Biobank (N\&equals; 112 151). Molecular Psychiatry 2016; 21: 758-67.

77. Huang J, Zhu T, Qu Y, Mu D. Prenatal, Perinatal and Neonatal Risk Factors for Intellectual Disability: A Systemic Review and Meta-Analysis. PloS one 2016; 11(4): e0153655.

78. Singh T, Kurki MI, Curtis D, Purcell SM, Crooks L, McRae J et al. Rare loss-of-function variants in SETD1A are associated with schizophrenia and developmental disorders. Nature neuroscience 2016; 19: 571-7.

79. De Rubeis S, He X, Goldberg AP, Poultney CS, Samocha K, Cicek AE et al. Synaptic, transcriptional and chromatin genes disrupted in autism. Nature 2014; 515(7526): 209-215.

80. Li J, Cai T, Jiang Y, Chen H, He X, Chen C et al. Genes with de novo mutations are shared by four neuropsychiatric disorders discovered from NPdenovo database. Molecular psychiatry 2015; 21: 298.

81. Sun L, Dimitromanolakis A. PREST-plus identifies pedigree errors and cryptic relatedness in the GAW18 sample using genome-wide SNP data. BMC proceedings 2014; 8(Suppl 1): S23I.

82. Riazuddin S, Hussain M, Razzaq A, lqbal Z, Shahzad M, Polla D et al. Exome sequencing of Pakistani consanguineous families identifies 30 novel candidate genes for recessive intellectual disability. Molecular psychiatry 2016 [Epub ahead of print]

83. Philips AK, Pinelli M, de Bie Cl, Mustonen A, Määttä T, Arts HH et al. Identification of C12orf4 as a gene for autosomal recessive intellectual disability. Clinical Genetics 2016; 91: 100-105. 
84. Alazami AM, Patel N, Shamseldin HE, Anazi S, Al-Dosari MS, Alzahrani F et al. Accelerating novel candidate gene discovery in neurogenetic disorders via whole-exome sequencing of prescreened multiplex consanguineous families. Cell reports 2015; 10(2): 148-161.

85. Vasli N, Ahmed I, Mittal K, Ohadi M, Mikhailov A, Rafiq MA et al. Identification of a homozygous missense mutation in LRP2 and a hemizygous missense mutation in TSPYL2 in a family with mild intellectual disability. Psychiatric genetics 2016; 26(2): 66-73.

86. Garshasbi M, Hadavi V, Habibi H, Kahrizi K, Kariminejad R, Behjati F et al. A defect in the TUSC3 gene is associated with autosomal recessive mental retardation. The American Journal of Human Genetics 2008; 82(5): 1158-1164.

87. Celis K, Shuldiner S, Haverfield EV, Cappell J, Yang R, Gong D-W et al. Loss of function mutation in glutamic pyruvate transaminase 2 (GPT2) causes developmental encephalopathy. Journal of inherited metabolic disease 2015; 38(5): 941-948.

88. Caldovic L, Morizono H, Panglao MG, Cheng SF, Packman S, Tuchman M. Null mutations in the Nacetylglutamate synthase gene associated with acute neonatal disease and hyperammonemia. Human genetics 2003; 112(4): 364-368.

89. Kutsche K, Yntema H, Brandt A, Jantke I, Nothwang HG, Orth U et al. Mutations in ARHGEF6, encoding a guanine nucleotide exchange factor for Rho GTPases, in patients with X-linked mental retardation. Nature genetics 2000; 26(2): 247-250.

90. Amir RE, Van den Veyver IB, Wan M, Tran CQ, Francke U, Zoghbi HY. Rett syndrome is caused by mutations in X-linked MECP2, encoding methyl-CpG-binding protein 2. Nature genetics 1999; 23(2): 185-188 


\section{Figure Legends:}

\section{Figure 1. Microarray and exome sequencing work flow.}

Figure 2. Pedigrees and HomozygosityMapper output for eight of the families. The locus harbouring the mutation is indicated in the HomozygosityMapper plots with yellow shading. HBD regions for each family, confirmed as autozygous and haploidentical, are provided in Supplementary Table 2. The gene name and mutation (at the protein level) are indicated, as well as genotypes for available family members. Additional pedigrees and HBD plots are provided in Supplementary Figure 2.

\section{Figure 3. Spatiotemporal expression of ID genes in human brain development: a) Scaled} expression trajectories of the ID Genes, averaged across brain regions. Solid lines are average expression for a group of genes. The lighter and thinner lines show trajectories of individual genes. Average expression for each gene group is depicted with points (green: glycosylationassociated (14 genes), blue: hormone or metabolic (20), purple: remainder of the ID Genes (66) and red: remaining genes assayed). Local regression was used to smooth the z-scored expression values for individual genes and gene group averages (LOESS). A vertical dashed line marks birth. b) Heatmap showing brain samples enriched for specific expression of ID genes. For each brain region and brain combination, the z-scored expression values for ID genes were compared against all other genes (Wilcoxon one-sided test, FDR-corrected p-values).

Significance levels are indicated by black (non-significant result), red $(p<0.05)$, orange $(p<$ $0.005)$ and yellow $(p<0.0005)$. Missing values are shown in grey. Brain regions include the ventrolateral prefrontal cortex (VFC), dorsolateral prefrontal cortex (DFC), orbital frontal cortex (OFC), anterior (rostral) cingulate (medial prefrontal) cortex (MFC), posterior (caudal) superior 
temporal cortex (area 22c) (STC), inferolateral temporal cortex (area TEv, area 20) (ITC), posteroventral (inferior) parietal cortex (IPC), primary motor cortex (area M1, area 4) (M1C), primary somatosensory cortex (area S1, areas 3,1,2) (S1C), primary auditory cortex (core) (A1C), primary visual cortex (striate cortex, area V1/17) (V1C), hippocampus (hippocampal formation) (HIP), amygdaloid complex (AMY), mediodorsal nucleus of thalamus (MD), striatum (STR) and cerebellar cortex (CBC).

Figure 4. Cross-disorder overlap: The Venn diagrams shown indicate genes for which a. variants have been reported in other neuropsychiatric or neurodevelopmental disorders, either homozygous, compound heterozygous, or de novo, through searches of published gene lists, including databases such as EpilepsyGene, Gene2Phenotype, Gene2Cognition, Schizophrenia Genebook, and published disease-specific gene sets ${ }^{6}, \mathrm{HGMD}^{28}$, and $\mathrm{OMIM}^{29} ;$ b. functional or animal models with relevant phenotypes have been reported, including resources such as $\mathrm{Zfin}^{30}$, targets of FMRP ${ }^{31}$ and Mouseportal (http://www.sanger.ac.uk/science/collaboration/mouse-resource-portal, accessed May 2016). Genes newly reported here are in red text. 
Table 1: Summary statistics for the Iranian and Pakistani family cohorts.

\begin{tabular}{|c|c|c|c|c|c|c|c|}
\hline $\begin{array}{c}\text { Source } \\
\text { Country }\end{array}$ & $\begin{array}{c}\text { Total } \\
\text { Families }\end{array}$ & $\begin{array}{c}\text { Total DNA } \\
\text { Samples }\end{array}$ & $\begin{array}{c}\text { Total } \\
\text { Affected }\end{array}$ & $\begin{array}{c}\text { Mean Number } \\
\text { of ID } \\
\text { Affected/Family }\end{array}$ & $\begin{array}{c}\text { F Coefficient } \\
\text { of } \\
\text { Inbreeding }\end{array}$ & $\begin{array}{c}\text { Number of Families with } \\
\text { Gene/Mutation } \\
\text { Identified: ARID (XLID; de } \\
\text { novo) }\end{array}$ & $\begin{array}{c}\text { Number of Families } \\
\text { with Pathogenic CNV } \\
\text { Identified }\end{array}$ \\
\hline Iran & 16 & 193 & 58 & 4.375 & 0.058034056 & $6(0 ; 0)$ & 0 \\
\hline Pakistan & 176 & 1379 & 627 & 3.5625 & 0.084862578 & $81(6 ; 1)$ & $9^{a}$ \\
\hline
\end{tabular}

${ }^{\mathrm{a}}$ In family PK73 two different likely pathogenic CNV losses were identified but are counted here as a single family with pathogenic CNV. 
Table 2: Novel candidate genes and sequence variants identified in the Pakistani and Iranian ID families. A list of all variants identified including known syndromic ARID or XLID genes identified in the cohort is given in Supplementary Table 3a; Supplementary Table 3b lists families in which between 2 and 4 variants were identified.. See Supplementary Table 3 for in silico predictions of the effects of the variants.

\begin{tabular}{|c|c|c|c|c|}
\hline & Family & Gene & Genome (ExAC MAF/S. Asian MAF) & cDNA (Protein) \\
\hline 1 & PJ7 & LAMC1 & Chr1:183083732A $>\mathrm{T}(0 / 0)$ & NM_002293.3:c.1088A>T (p.His363Leu) \\
\hline 2 & PK113 & AFF3 & Chr2:100167973C>A (0/0) & NM_002285.2:c.3644G>T (p.Gly1215Val) \\
\hline $3^{a}$ & AS61 & $A B / 2$ & Chr2:204245039C>T (0/0) & NM_005759.4:c.394C>T (p.Arg132*) \\
\hline $4^{b}$ & PK34 & UBA7 & Chr3:49848458C>A (8.01E-04/5.45E-03) & NM_003335.2:c.1189G>T (p.Glu397*) \\
\hline 5 & PJ9 & $T B C 1 D 23$ & Chr3:100037953delA (0/0) & NM_018309.4:c.1683delA (p.Glu562Argfs*3) \\
\hline 6 & AN50 & ZBTB11 & Chr3:101371344A>C (0/0) & NM_014415.3:c.2640T>G (p.His880GIn) \\
\hline 7 & AS70 & MAP3K7 & Chr6:91254333C>T (1.65E-05/1.21E-04) & NM_145331.2:c.1229G>A (p.Arg410GIn) \\
\hline 8 & PJ2 & SUMF2 & Chr7:56146063A>G (8.70E-06/7.73E-5) & NM_015411.2:c.740A>G (p. Tyr247Cys) \\
\hline 9 & AS20 & EXTL3 & Chr8:28,575,513C>G (0/0) & NM_001440.3:c.1937C>G (p. Ser646Cys) \\
\hline $10^{c}$ & AS66 & MPDZ & Chr9:13109992_13109995delGAAA (0/0) & NM_003829.4:c.5811_5814delTTTC (p.Phe1938Leufs*3) \\
\hline 11 & AS22 & MAPK8 & Chr10:49628265_49628265delC (0/0) & NM_002750.3:c.518delC (p.Arg174Glyfs*8) \\
\hline 12 & PK70 & TET1 & Chr10:70451328G>T (0/0) & NM_030625.2:c.6168G>T (p.Lys2056Asn) \\
\hline 13 & IDSG19 & DMBT1 & Chr10:124356559C>G (0/na) & NM_007329.2:c.2906C>G (p.Thr969Arg) \\
\hline $14^{\mathrm{d}}$ & AS105 \& 110 & PIDD1 & Chr11:799453G>A (0/0) & NM_145886.3:c.2587C>T (p.Gln863*) \\
\hline 15 & PK135 & $B D N F$ & Chr11:27679747A>G (1.65E-04/1.21E-03) & NM_001709.4:c.365T>C (p.Met122Thr) \\
\hline 16 & PK94 & USP44 & Chr12:95927147_95927160delinsA (0/0) & NM_032147.3:c.873_886delinsT (p.Leu291Phefs*8) \\
\hline 17 & AS23 & SPATA13 & Chr13:24797332C>T (4.71E-05/0) & NM_001166271.1:c.265C>T (p.Arg89Trp) \\
\hline 18 & PK68 & SLAIN1 & Chr13:78320722_78320722delA (0/0) & NM_144595.3:c.135delA (p.Thr49Hisfs*96) \\
\hline 19 & IDH3 & TRAPPC6B & Chr14:39628712G>A (1.65E-05/na) & NM_177452.3:c.124C>T (p.Arg42*) \\
\hline 20 & PK79 & VPS35 & Chr16:46705735T>G (0/0) & NM_018206.4:c.1406A>C (p.Gln469Pro) \\
\hline 21 & PK91 & SYNRG & Chr17:35896199C>T (8.66E-06/0) & NM_007247.4:c.3548G>A (p.Arg1183His) \\
\hline 22 & IDSG29 & FBX047 & Chr17:37107906G>C (0/na) & NM_001008777.2:c.544C>G (p.Arg182Gly) \\
\hline 23 & AN53 & SDK2 & Chr17:71431658C>T (5.82E-05/0) & NM_001144952.1:c.1126G>A (p.Gly376Ser) \\
\hline 24 & AN48 & CAPS & Chr19:5914970G>A (2.17E-04/1.27E-04) & NM_004058.3:c.281G>A (p.Arg94GIn) \\
\hline 25 & PJ6 & GPR64 & ChrX:19055718T>C (8.45E-05/0) & NM_005756.3:c.191A>G (p.Asn64Ser) \\
\hline 26 & PK87 & MAGEA11 & ChrX:148796213C>T (0/0) & NM_005366.4:c.169T>C (p.Ser57Pro) \\
\hline
\end{tabular}

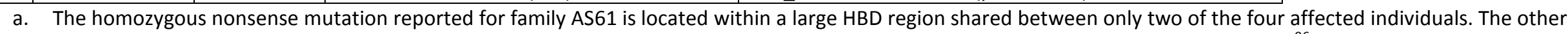
two are phenotypically distinct with ID and myopathy. Support for this gene comes from functional studies, including with $A b i 2$ null mice ${ }^{96}$.

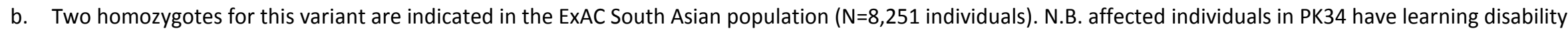
rather than ID. The South Asian cohort used in ExAC was part of the Pakistan Risk of Myocardial Infarction Study (PROMIS) and would not have excluded subjects in this category.

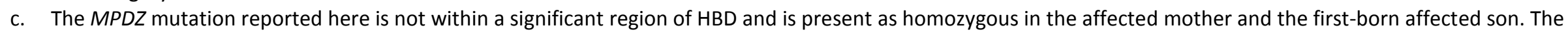
affected brother and sister are both heterozygous, but the father is wild type homozygous, thus suggesting non paternity for the first child.

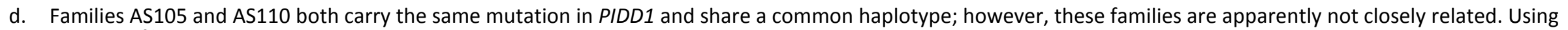
Prest-plus ${ }^{81}$ to estimate IBD using Maximum Likelihood Estimation, it was confirmed that the two families are separated by at least four generations, which is 
comparable to relatedness in the background population, and thus AS105 and AS110 are considered unrelated. na=not applicable, because Iranian exome data are not available in the ExAC database. 
Table 3: Candidate genes and sequence variants identified in the Pakistani and Iranian ID families in support of previously reported nonsyndromic ID genes. Asterisks indicate genes/variants that we have previously reported in this cohort. For subjects in whom the phenotype was clearly NS-ARID, despite prior association of the gene with S-ARID (e.g. LRP2 and MECP2), we have included the variant in this list. See Table S3 for in silico predictions of the effects of the variants.

\begin{tabular}{|c|c|c|c|c|c|c|}
\hline & Family & Gene & Disease; MIM \# & Genome (ExAC MAF/S. Asian MAF) & cDNA (Protein) & Ref \\
\hline 1 & AN49 & FMN2* & MRT47; 616193 & Chr1:240370627_240370628delinsG (0/0) & NM_020066.4:c.2515_2517delinsCG (p.Thr839Argfs*48) & 16 \\
\hline $2^{a}$ & AS114 & FMN2 & MRT47; 616193 & Chr1:240370641_240370642delCT (0/0) & NM_020066.4: c.2529_2530delCT (p.Ser844Cysfs*6) & \\
\hline 3 & PJ12 & LRP2* & 222448 & Chr2:170027106C >T (2.47E-04/9.08E-04) & NM_004525.2: c.11335G>A (p.Asp3779Asn) & 85 \\
\hline 4 & IDH10 & $H N M T^{*}$ & MRT51; 616739 & Chr2:138727776G>A (1.66E-05/na) & NM_006895.2:c.179G>A (p.Glu60Asp) & 18 \\
\hline $5^{b}$ & PK11 & TUSC3 & MRT7; 611093 & Chr8:15480694C>T (0/0) & NM_006765.3:c.244C>T (p.Arg82*) & 86 \\
\hline 6 & AN21 & TUSC3* & MRT7: 611093 & Chr8:15,521,688_15,692,362del (N/A) & NM_178234 del exons 5-10 & 78 \\
\hline 7 & PK54 & IMPA1 & & Chr8:82583195_82583196delinsC (0/0) & NM_005536.3:c.544_545delinsG (p.Leu182Valfs*54) & 45 \\
\hline 8 & AS19 & TRAPPC9* & MRT13; 613192 & Chr8:141407724G>A (8.24E-06/6.06E-05) & NM_031466.7:c.1423C>T (p.Arg475*) & 13 \\
\hline 9 & AS102 & PGAP2 & MRT17; 614207 & Chr11:3846254G>C (4.95E-05/3.64E-04) & NM_014489.3:c.713G>C (p.Arg238Pro) & 42 \\
\hline 10 & PK62 & TMEM135 & & Chr11:87032300delTT (0/0) & NM_022918.3:c.1304_1305delTT (p.Phe435Serfs*31) & 12 \\
\hline 11 & AS17 & CCDC82 & & Chr11:96117377G>A (1.65E-05/6.05E-05) & NM_024725.3:c.535C>T (p.Arg179*) & 82 \\
\hline 12 & ZA5 & C12ORF4 & & Chr12:4599717delA (0/0) & NM_020374.2:c.1537delT (p.Ser513Leufs*7) & 83,84 \\
\hline 13 & PJ5 & DCPS* & ARS; 616459 & Chr11:126208295G>A (8.25E-06/0) & NM_014026.3: c.636+1G>A (15 amino acid insertion) & 17 \\
\hline $14^{\mathrm{c}}$ & PK12 & GPT2 & MRT49; 616281 & Chr16:46918865C>G (0/0) & NM_133443.2:c.238C>G (p.Gln80Glu) & 87 \\
\hline 15 & PK85 & GPT2 & MRT49; 616281 & Chr16:46956326C>T (3.33E-05/1.89E-04) & NM_133443.3:c.1210C>T (p.Arg404*) & 87 \\
\hline 17 & PK33 & NAGS & NAGSD; 237310 & Chr17:42085083C>T (7.74E-05/4.54E-04) & NM_153006.2:c.1393C>T (p.Arg465Trp) & 88 \\
\hline 18 & PK31 & METTL23* & MRT44; 615942 & Chr17:74729449C>T (0/0) & NM_001080510:c.397C>T (p.Gln133*) & 15 \\
\hline 19 & IAID3 & METTL23* & MRT44; 615942 & Chr17: 74729211_74729215del (0/0) & NM_001080510.3:c.236_240del (p.Thr80Glyfs*20) & 15 \\
\hline 20 & AN37 & TRMT1 & & Chr19:13223779_13223810del (0/0) & NM_017722.3:c.657_688del (p.Gln219Hisfs*22) & 12 \\
\hline 21 & PJ3 & MBOAT7 & & Chr19:54684518_54684524del (0/0) & NM_024298.3:c.820_826del (p. Gly274Profs*47) & 35 \\
\hline 22 & AS101 & ARHGEF6 & MRX46; 300436 & ChrX:135829705A>G (1.14E-05/9.91E-05) & NM_004840.2:c.296T>C (p.Val99Ala) & 89 \\
\hline 23 & PK55 & MECP2 & MRXS13; 300055 & ChrX:153296710C>T (0/0) & NM_004992.3:c.569G>A (p.Arg190His) & 90 \\
\hline
\end{tabular}

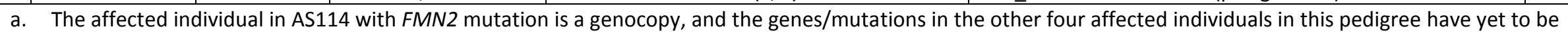
established.

b. The homozygous nonsense mutation in TUSC3 is not in a shared HBD region and does not segregate with other affected members of family PK11.

c. The missense change in GPT2 is also predicted to affect a splice donor site (BDGP (www.fruitfly.org) splice predictor score: WT: 0.78 ; mut: 0.58).

na=not applicable, as Iranian exome data are not available in the ExAC database. 
Supplementary Information:

Supplementary Methods

Supplementary Figure 1: Bioinformatic pipeline for whole exome sequence (WES) analysis.

Supplementary Figure 2: Pedigrees, HomozygosityMapper output and FSuite circo-plots for families with single variants identified, in addition to those shown in Figure 2.

Supplementary Figure 3: Spatiotemporal expression of ID genes in human development using RNA sequencing data.

Supplementary Figure 4: Developmental expression pattern of ID genes in the human prefrontal cortex.

Supplementary Table 1: Family statistics

Supplementary Table 2: Homozygosity-by-descent/autozygosity shared regions, as defined using HomozygosityMapper, cross-referenced with FSuite.

Supplementary Table 3: Mutations identified per family. A. Single homozygous variant identified. B. Two to four variants identified. C. Dominant/de novo mutation identified.

Supplementary Table 4: Pathogenic CNVs and variants of unknown significance identified by microarray analysis.

Supplementary Table 5: BioGRID protein interaction analysis. 
Supplementary Table 6: Gene Ontology Pathway analysis

Supplementary Table 7: Gene List for anatomic/temporal transcription analyses.

Supplementary Table 8: Top anatomical regions for ID gene expression. 


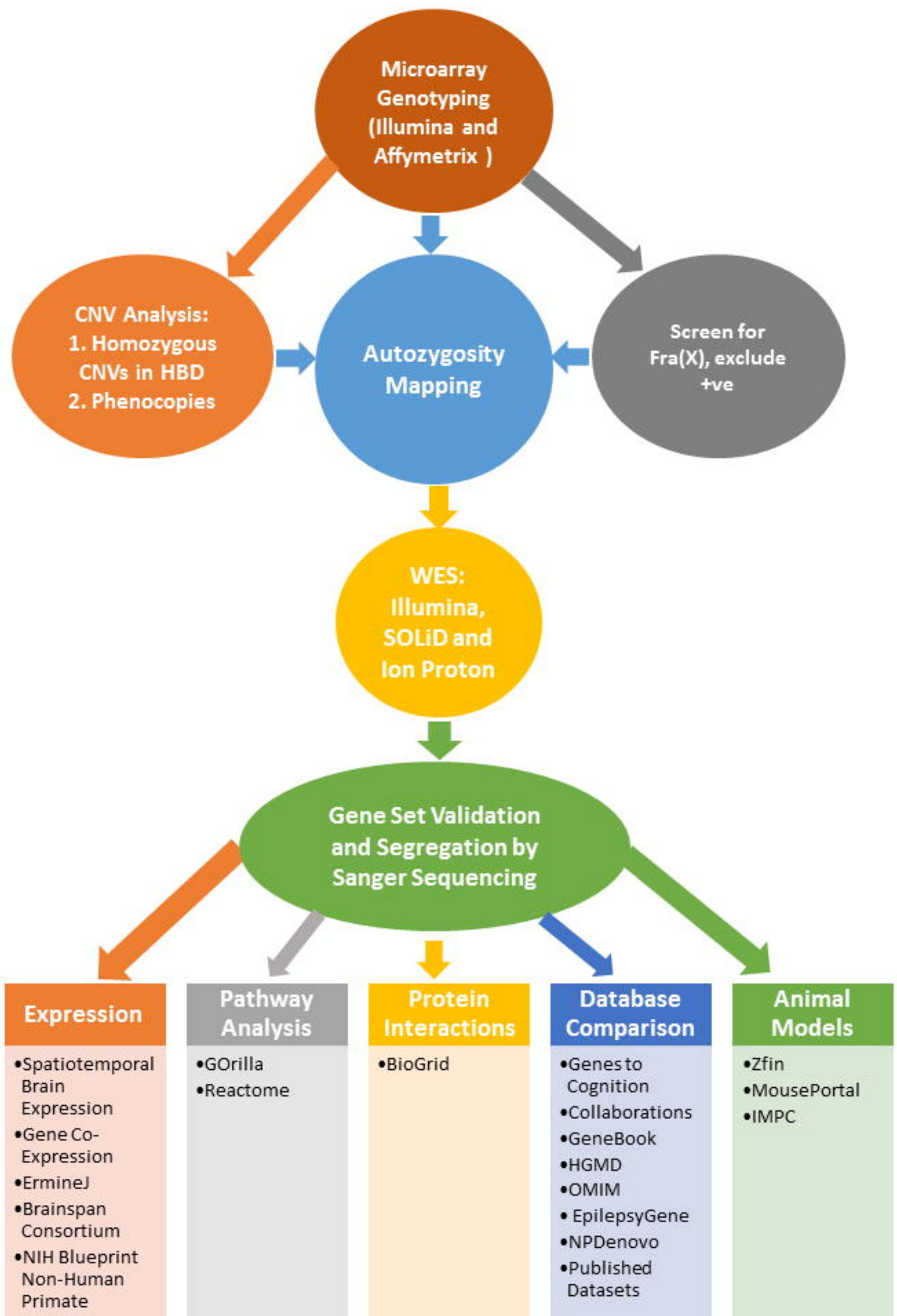



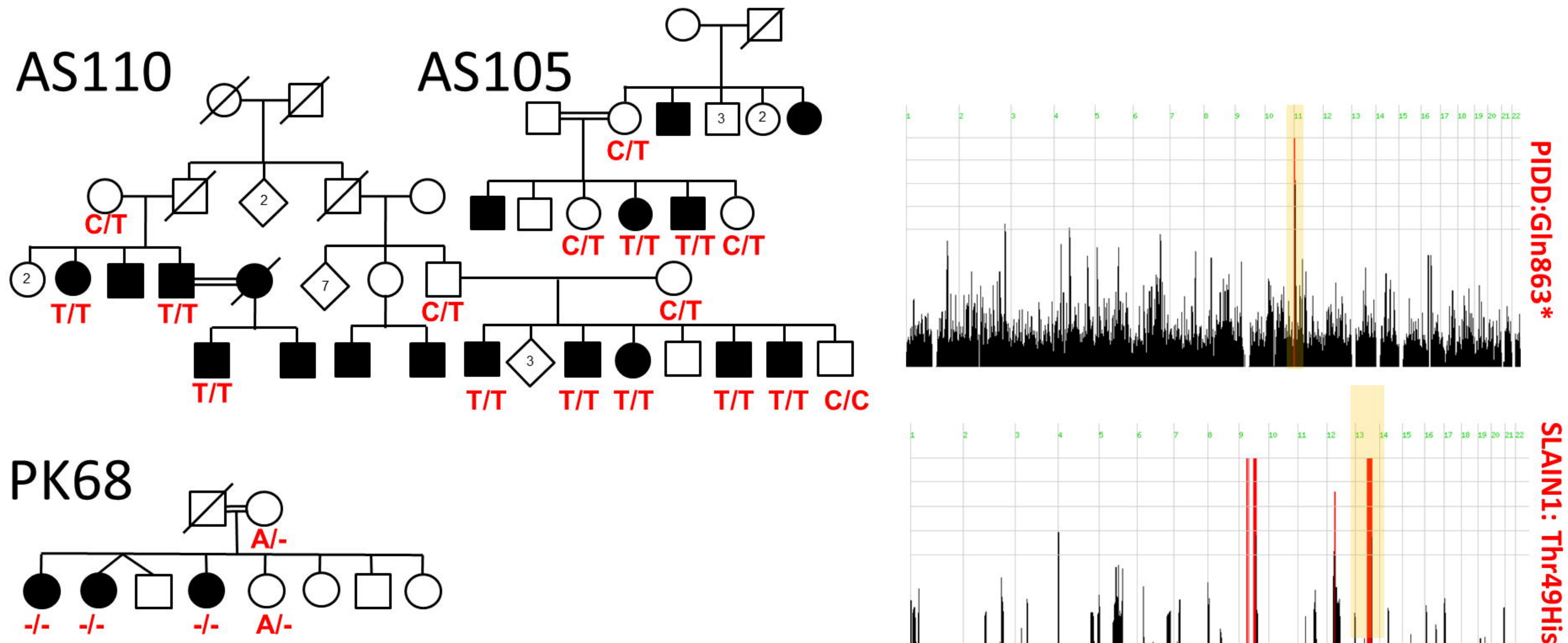

PK135
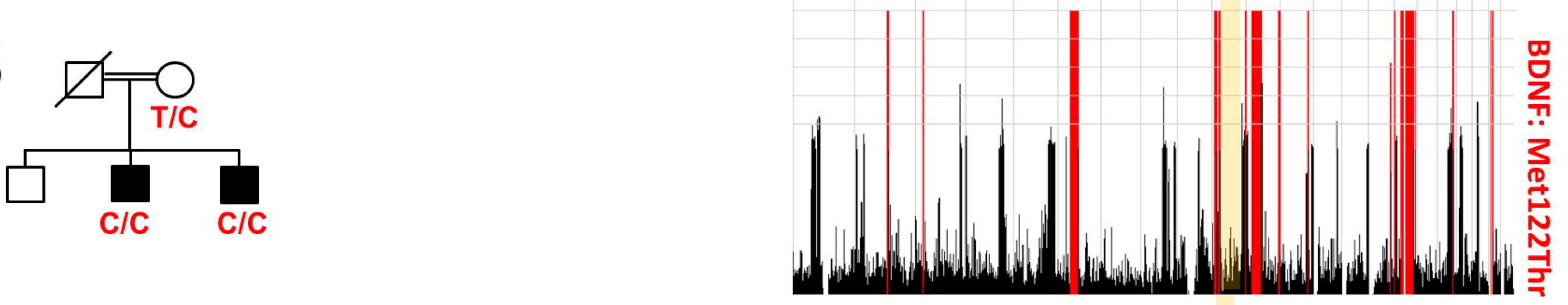

PK70

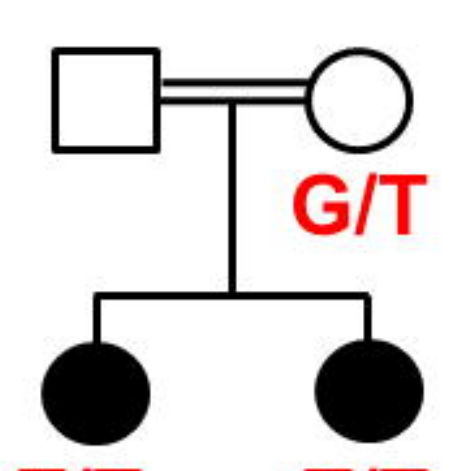

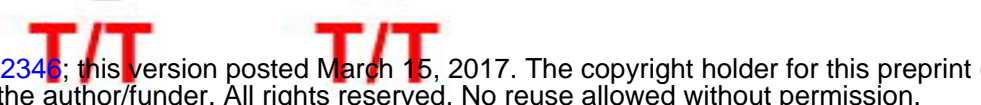

PJ9

$\triangle \varnothing$
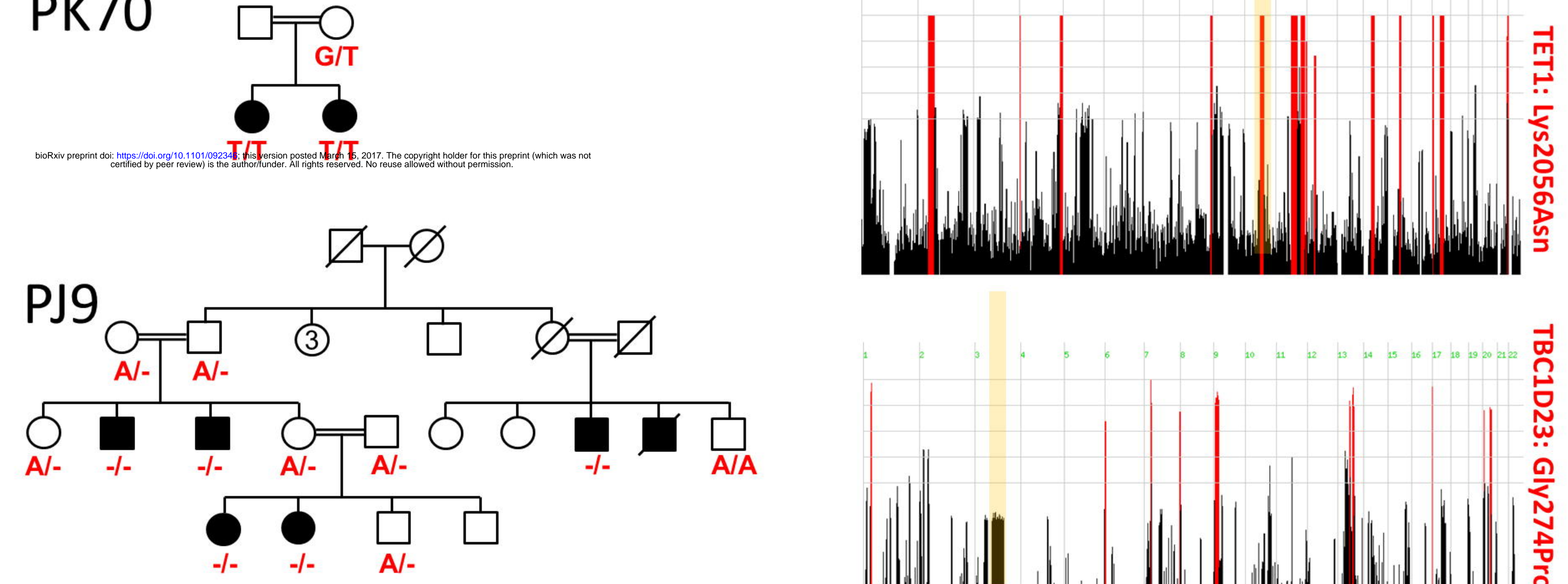

AN50 $\square$
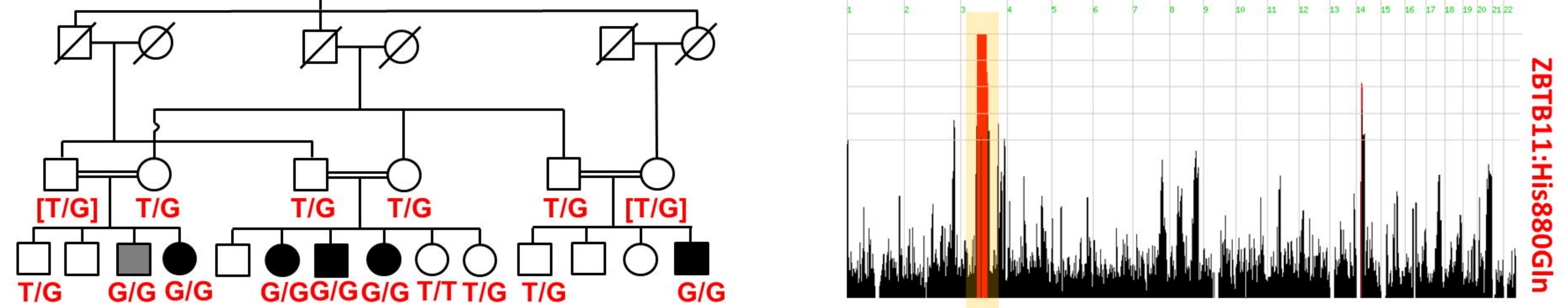


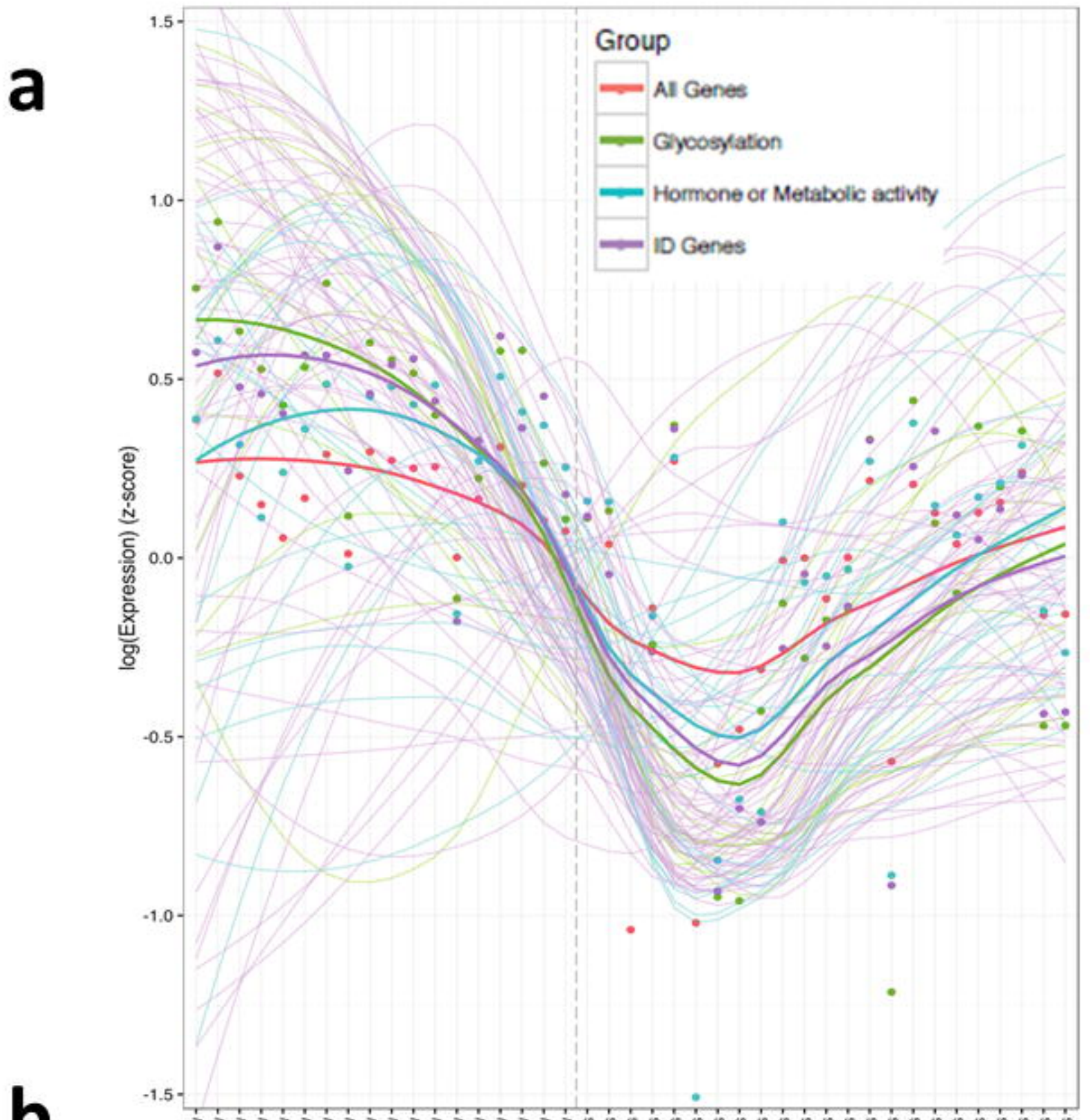

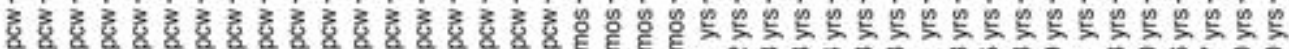

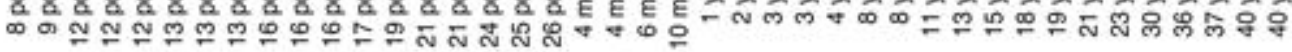

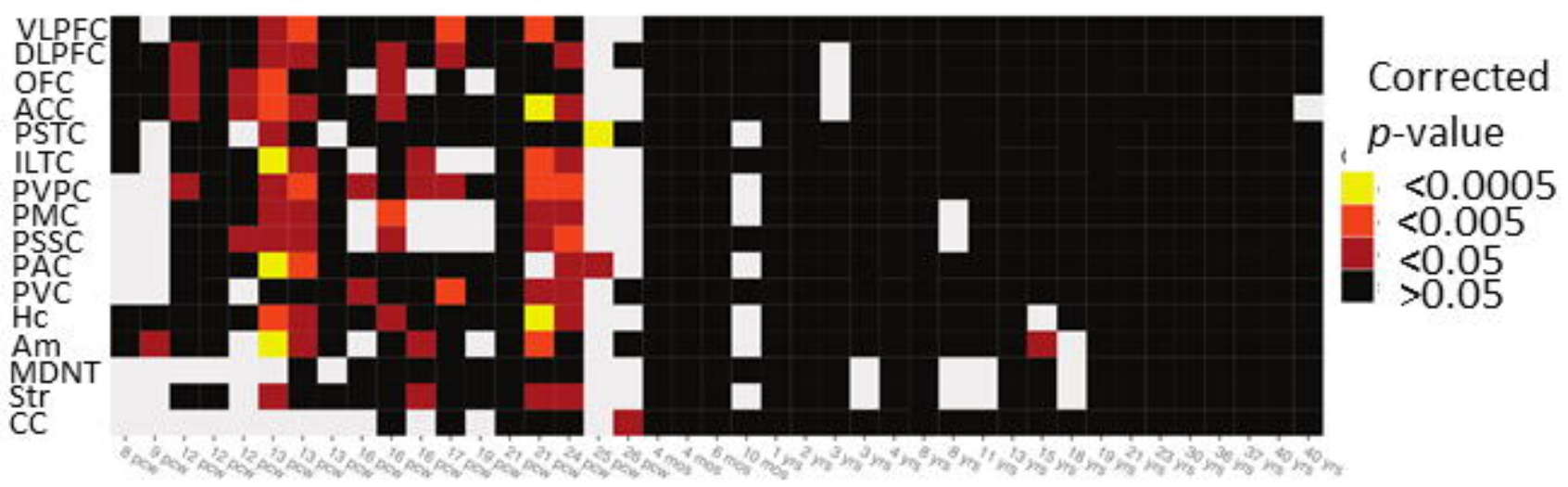




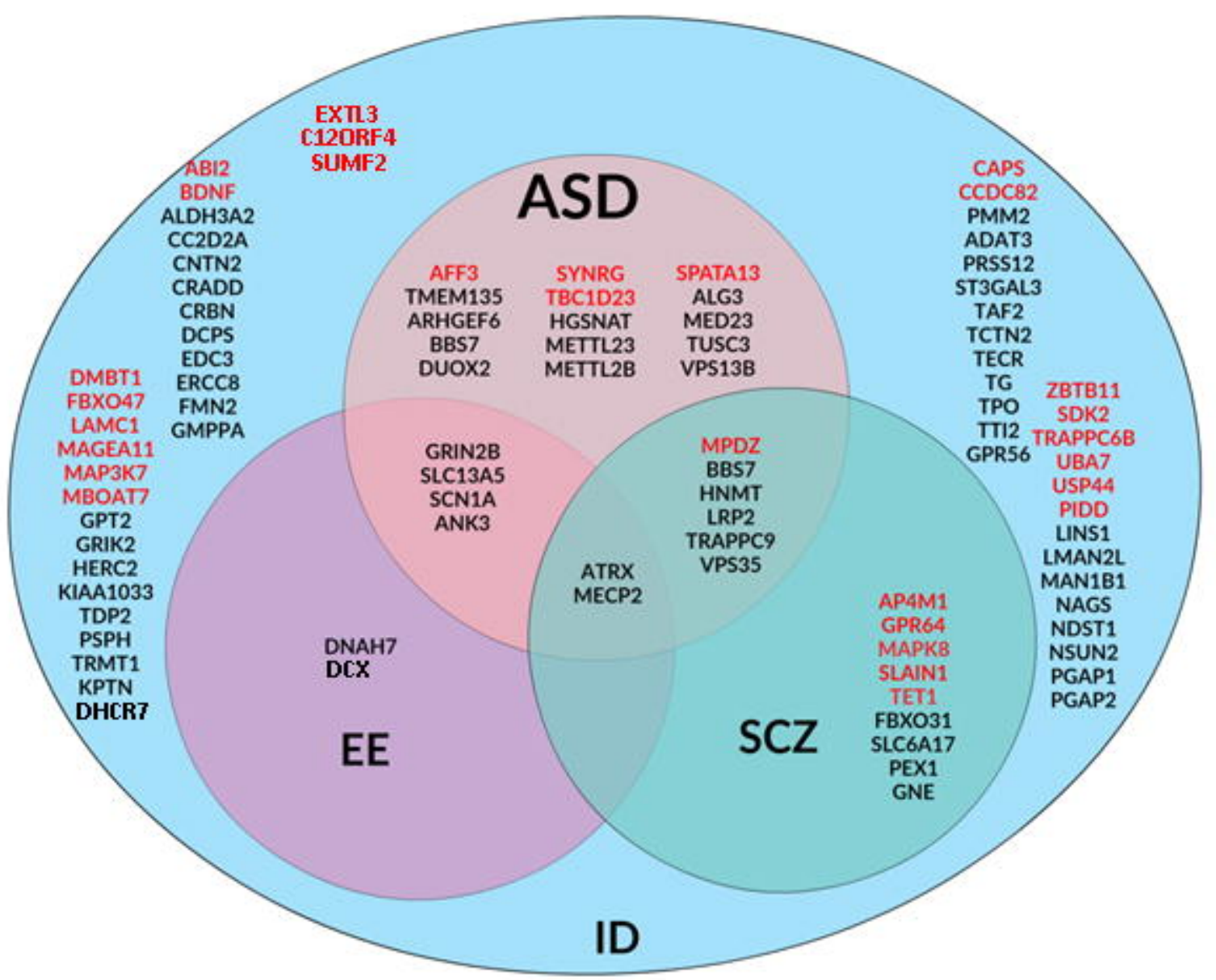

b

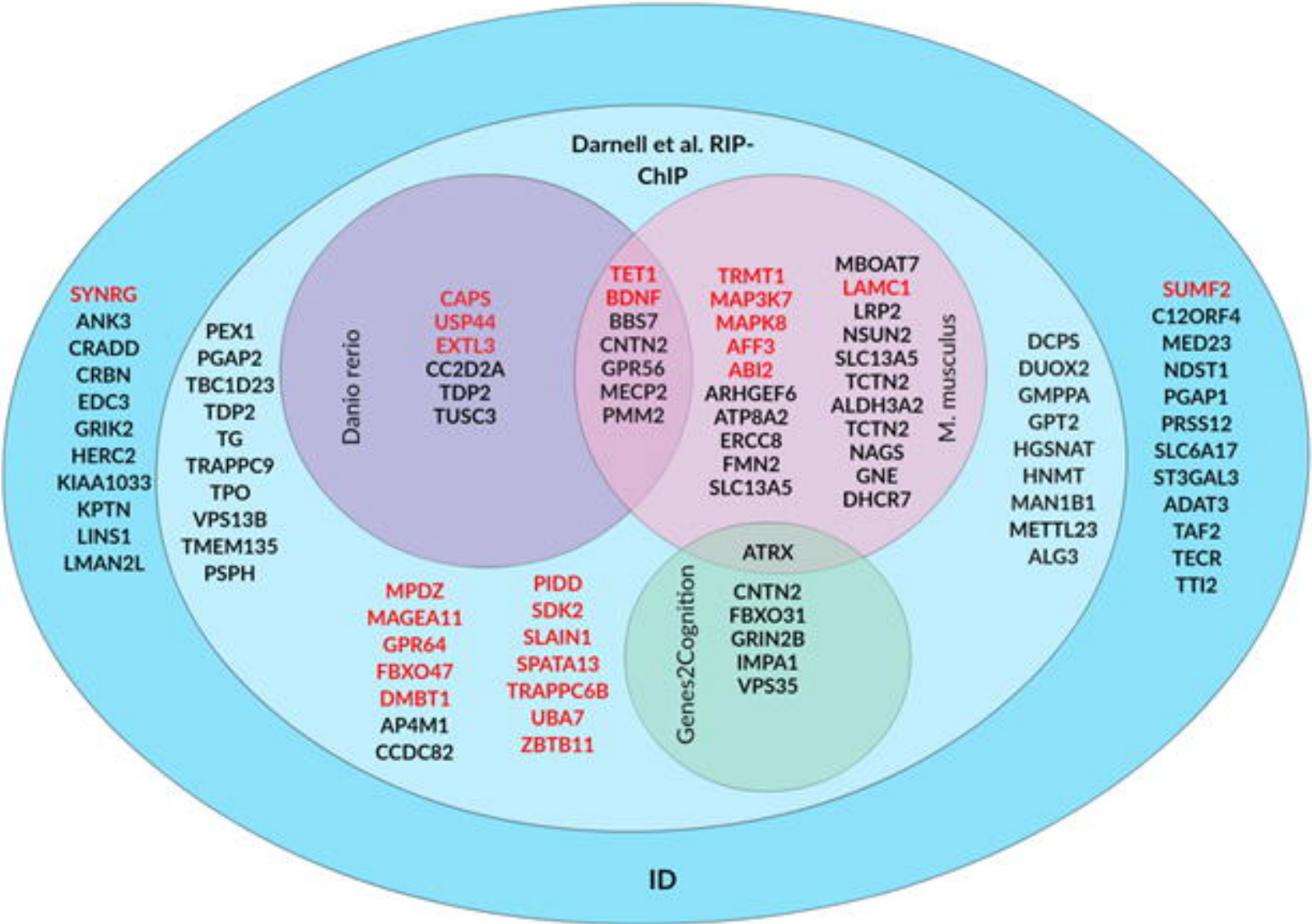

